\title{
Quality performance measures for small capsule endoscopy: Are the ESGE quality standards met?
}

\section{(ㄷ)(1) $\odot$}

\section{Authors}

Emanuele Rondonotti ${ }^{*}, 1$, Cristiano Spada*, 2,3, Sergio Cadoni ${ }^{4}$, Renato Cannizzaro ${ }^{5}$, Carlo Calabrese ${ }^{6}$, Roberto de Franchis $^{7}$, Luca Elli ${ }^{8}$, Carlo Maria Girelli ${ }^{9}$, Cesare Hassan ${ }^{10}$, Riccardo Marmo ${ }^{11}$, Maria Elena Riccioni ${ }^{12}$, Salvatore Oliva $^{13}$, Giuseppe Scarpulla ${ }^{14}$, Marco Soncini ${ }^{15}$, Maurizio Vecchi ${ }^{16}$, Marco Pennazio ${ }^{17}$, “RAVE Study Group”

\section{Institutions}

1 Unità Operativa Complessa di Gastroenterologia, Ospedale Valduce, Como, Italy

2 Unità Operativa di Endoscopia Digestiva, Università Cattolica, Rome, Italy

3 Unità Operativa di Endoscopia Digestiva, Fondazione Poliambulanza, Brescia, Italy

4 Unità Operativa di Endoscopia Digestiva, Centro Traumatologico Ortopedico, Iglesias, Italy

5 Struttura Operativa Complessa di Gastroenterologia Oncologica, Centro di Riferimento Oncologico di Aviano (CRO), Istituto Nazionale Tumori IRCCS, Aviano, Italy

6 Unità Operativa Malattia Infiammatorie Croniche Intestinali, Dipartimento di Medicina e Chirurgia (DIMEC), Ospedale S. Orsola-Malpighi Università di Bologna, Bologna, Italy

7 Università degli Studi di Milano, Milan, Italy

8 Unità Operativa Complessa di Gastroenterologia ed Endoscopia-Centro per la Prevenzione e Diagnosi della Malattia Celiaca, Fondazione IRCCS Ca' Granda Ospedale Maggiore Policlinico, Milan, Italy

9 Unità Di Gastroenterologia ed Endoscopia Digestiva, Ospedale di Busto Arsizio, Busto Arsizio, Italy

10 Unità Operativa di Endoscopia Digestiva, Ospedale Nuovo Regina, Rome, Italy

11 Unità Operativa di Gastroenterologia, Ospedale Curto, Polla, Italy

12 Unità Operativa di Endoscopia Digestiva, IRCCS Fondazione Policlinico Universitario A. Gemelli, Rome, Italy

13 Unità Operativa di Gastroenterologia e Epatologia Pediatrica, Università La Sapienza, Rome, Italy

14 Unità Operativa di Gastroenterologia, Ospedale M. Raimondi, San Cataldo, Italy

15 Dipartimento di Medicina Interna, Ospedale Alessandro Manzoni, Lecco, Italy

16 Dipartimento di Scienze Biomediche, Università degli Studi di Milan, Fondazione IRCCS Ca' Granda Ospedale Maggiore Policlinico, Milan, Italy
17 Divisione di Gastroenterologia U, Azienda OspedalieroUniversitaria, Città della Salute e della Scienza, Torino, Italy

submitted 16.5 .2020

accepted after revision 19.10 .2020

Bibliography

Endoscopy International Open 2021; 09: E122-E129

DOI 10.1055/a-1319-0742

ISSN 2364-3722

(c) 2021. The Author(s).

This is an open access article published by Thieme under the terms of the Creative Commons Attribution-NonDerivative-NonCommercial License, permitting copying and reproduction so long as the original work is given appropriate credit. Contents may not be used for commecial purposes, or adapted, remixed, transformed or built upon. (https://creativecommons.org/licenses/by-nc-nd/4.0/)

Georg Thieme Verlag KG, Rüdigerstraße 14,

70469 Stuttgart, Germany

Corresponding author Emanuele Rondonotti MD, PhD, Gastroenterology Unit, Ospedale Valduce, Via Dante 11, 22100, Como, Italy Fax: +0039031324150

ema.rondo@gmail.com

Supplementary material is available under https://doi.org/10.1055/a-1319-0742

\section{ABSTRACT}

Background and study aims The European Society of Gastrointestinal Endoscopy (ESGE) recently issued a quality performance measures document for small bowel capsule endoscopy (SBCE). The aim of this nationwide survey was to explore SBCE practice with ESGE quality measures as a benchmark.

Patients and methods A dedicated per-center semiquantitative questionnaire based on ESGE performance measures for SBCE was created by a group of SBCE experts. One-hundred-eighty-one centers were invited to partici-

\footnotetext{
* These authors contributed equally
} 
pate and were asked to calculate performance measures for SBCE performed in 2018. Data were compared with 10 ESGE quality standards for both key and minor performance measures.

Results Ninety-one centers (50.3\%) participated in the data collection. Overall in the last 5 years (2014-2018), 26,615 SBCEs were performed, 5917 of which were done in 2018. Eighty percent or more of the participating centers reached the minimum standard established by the ESGE Small Bowel Working Group (ESBWG) for four performance measures (indications for SBCE, complete small bowel evaluation, diagnostic yield and retention rate). Conversely, compliance with six minimum standards established by ESBWG concerning adequate bowel preparation, patient selection, timing of SBCE in overt bleeding, appropriate reporting, reading protocols and referral to device-assisted enteroscopy was met by only $15.5 \%, 10.9 \%, 31.1 \%, 67.7 \%$, $53.4 \%$, and $32.2 \%$ of centers, respectively.

Conclusions The present survey shows significant variability across SBCE centers; only four (4/10: 40\%) SBCE procedural minimum standards were met by a relevant proportion of the centers ( $\geq 80 \%$ ). Our data should help in identifying target areas for quality improvement programs in SBCE.

\section{Introduction}

Variation in endoscopic quality was found to impact important health outcomes, which improve when physicians receive timely feedback and meet consensus threshold goals [1-5]. This led to substantial efforts to develop quality assurance policies and quality improvement programs [6-11]. The development of robust, consensus- and evidence-based key performance measures is the first step in any quality improvement program. Ideally, these measures should help physicians and services to identify, appraise, and monitor the key steps in the endoscopy-based care processes, determine if variability exists across centers exists and indicate where systems are suboptimal and whether the service is providing high-quality patient-centered healthcare [7].

To develop standards for high quality in gastrointestinal endoscopy, the European Society of Gastrointestinal Endoscopy (ESGE) has established the ESGE Quality Improvement Committee, which involved several gastrointestinal endoscopy experts to set up quality indicators [9-12] that, when met, reflect a high-quality examination for different endoscopic procedures. The ESGE Quality Improvement Committee recently appointed a Small Bowel Working Group (ESBWG) to identify a list of performance measures for small bowel endoscopy, including small bowel capsule endoscopy (SBCE) [13], which has unique technical features. The ESBWG identified six key quality performance measures and four minor performance measures. For each of them, a minimum standard and a target standard was established by consensus [13] ( Table 1).

To the best of our knowledge, at present, there are no studies in which SBCE performance has been compared to these quality standards. The aim of the present survey was to explore variability in SBCE performance in clinical practice with ESGE standards as a quality benchmark to provide a snapshot of the quality of Italian endoscopy SBCE services and to identify areas that might be targeted for quality improvement programs.

- Table 1 Summary of domains, performance measures, and standards (both minimum and target standards) for small bowel capsule endoscopy.

\begin{tabular}{|c|c|c|c|}
\hline Domain & Performance measure & Minimum standard & Target standard \\
\hline \multirow[t]{3}{*}{ Pre-procedure } & Indication for SBCE & $\geq 95 \%$ & $\geq 95 \%$ \\
\hline & Adequate bowel preparation & $\geq 95 \%$ & $\geq 95 \%$ \\
\hline & Patient selection & $\geq 95 \%$ & $\geq 95 \%$ \\
\hline Completeness of procedure & Caecal or stoma visualization & $\geq 80 \%$ & $\geq 95 \%$ \\
\hline \multirow[t]{4}{*}{ Identification of pathology } & Lesion detection rate & $\geq 50 \%$ & $\geq 50 \%$ \\
\hline & Timing of SBCE for overt bleeding & $\geq 90 \%$ & $\geq 90 \%$ \\
\hline & Use of standard terminology & $\geq 90 \%$ & $\geq 90 \%$ \\
\hline & Reading speed of SBCE & $\geq 90 \%$ & $\geq 95 \%$ \\
\hline Management of pathology & Appropriate referral for DAE & $\geq 75 \%$ & $\geq 90 \%$ \\
\hline Complications & Capsule retention rate & $<2 \%$ & $<2 \%$ \\
\hline \multicolumn{4}{|c|}{$\begin{array}{l}\text { Key performance measures are indication for SBCE, cecal or stoma visualization, lesion detection rate, timing of SBCE for overt bleeding, appropriate referral for DA } \\
\text { and capsule retention rate. The other performance measures are minor. } \\
\text { SBCE, small bowel capsule endoscopy; DAE, device-assisted enteroscopy. }\end{array}$} \\
\hline
\end{tabular}




\section{Material and methods}

In February 2019, a panel of 16 Italian gastroenterologists, including both capsule endoscopy experts (ER, MP, CS, RC, SC, CC, RdF, LE, CMG, RM, MER, SO, GS, MS and MV; all of them read $>500$ SBCEs and 7/15 also performed DAE on a routine basis) and quality assurance experts $(\mathrm{CH}, \mathrm{CS})$, created a dedicated questionnaire (Appendix 1), based on the SBCE performance measures. It was a per-center semi-quantitative multiplechoice questionnaire: no data on individual patients were collected, and intervals or ranges were reported when quantitative or numerical variables were concerned.

The questionnaire had four sections. Section A (14 items) was focused on center features (e.g., location, number of physicians performing SBCE, number of SBCE performed per year, etc.). Section A was intended to provide a snapshot of the diffusion of the SBCE technique over the country and over time; therefore, data about a broader timeframe (2014-2018) were collected. Section B (7 items) evaluated the current policy of centers toward the evaluation of quality indicators concerning both non-small bowel and small bowel endoscopic procedures before the publication of ESGE performance measures for small bowel endoscopy. In "Section C" (10 items) the questions mirrored the ESBWG document structure: a participating center was asked to measure each performance indicator, according to the description and the construct addressed in the ESGE document, and to report whether the ESGE standard (minimum and target) was met. To collect reliable data and to map the existing situation in Italy at the time of publication of the ESGE quality standards, the centers were asked to answer questions in "Section C," taking into account only SBCEs performed in 2018 by checking hospital databases and SBCE reports. Focusing on a definite and relatively short (12-month) timeframe allowed for collection of more reliable, solid, representative, and actual data. All the questions included in Section $C$ addressing performance measures were labeled as mandatory. At the end of Section $C$ questionnaire respondents who were willing to be involved in further research and/or quality improvement programs had the opportunity to identify themselves. Section D was optional; it included nine questions about procedural and practical issues.

In April 2019, the questionnaire was uploaded to a webbased platform. Through March 2019, only one physician per center was initially invited; if no answer was provided in 3 weeks, a second physician from the same center was then automatically invited. As a consequence, in some cases, more than one physician per center might have received the invitation email. To prevent data duplication, a strong disclaimer was included in the invitation e-mail, as well as in each questionnaire section, asking the recipient to provide only one dataset per center. Furthermore, to identify possible multiple entries from the same center, the structural data reported in Section A, along with the self-identification at the end of Section $C$, if provided, were matched on a regional basis. In case of suspected data duplication, only the latest uploaded dataset was included.
The web link reported above contained a numerical individual code that differed from center to center and allowed the system to automatically send periodic reminders, keeping the entire process fully anonymous. It was not possible for either the person sending the invitation or those who analyzed the data to identify the center or the physician filling the questionnaire, unless the respondent decided to identify himself or herself by completing Section $C$ last question. The questionnaire was available on the website for 6 months. During that time, automatic reminder e-mails were sent on a monthly basis. Collected data were presented and discussed in January 2020, during the Capsule Endoscopy Annual Meeting (Riunione Annuale Videocapsula Endoscopica-RAVE, January 24-25, Vimercate, Italy) to which at least one representative per center had been invited. Both gastroenterologists who completed the questionnaires and those who did not participated in the meeting: overall 110 gastroenterologists participated in the last RAVE meeting.

\section{Statistical analysis}

Information only from centers that answered more than $80 \%$ of questions, including all those labeled as mandatory was included in the data analysis. The data were collected, reviewed, and analyzed only by the experts who generated the questionnaire. Non-normally distributed data were presented using median and interquartile ranges (IQR). Comparisons of proportions were performed by Fisher's exact test or chi-square test (with Yates correction, if cell frequencies were below 10), as appropriate. For all comparisons, a $\mathrm{P} \leq 0.05$ (2-sided statistical hypothesis test) was considered statistically significant. The statistical analysis was carried out with Excel software (Office package V.2016; Microsoft Co, Redmonton, Washington, United States), or through the GraphpadQuickcalcs website, available online at www.graphpad.com/quickcalcs/contMenu/.

\section{Subanalyses}

Performance measures were stratified according to center type: for this purpose, centers were dichotomized as academic (including research hospitals and university hospitals) or nonacademic (including both public hospitals and private hospitals part of the "state-run" healthcare system; these institutions follow the same rules and the same reimbursement policy, as far as SBCEs procedures are concerned). Similarly, 2018 performance measures were also analyzed according to the 2018 caseload: centers performing $\leq 35$ SBCEs in 2018 were defined as low volume centers, whereas those performing $\geq 36$ SBCEs were defined as medium-high volume centres. Although we have to acknowledge that this threshold (35 procedures") is fully arbitrary, taking into account the available evidence [1317] the recently published ESGE Position Statement [18] stated that competence in SBCE can be assessed by considering a minimum of 30 SBCEs. Therefore, we might argue that 35 could represent a reasonable number of procedures to achieve and maintain proficiency in reading SBCE over time. Therefore, we defined as "low-volume" centers performing less than 35 examinations/year and "medium-high volume" those performing more than 35 SBCEs/year and. 


\section{Results}

\section{Center features}

Of 181 invited physicians, 106 (58.6\%) accessed the website and 91 of them answered more than $80 \%$ of questions, including all the mandatory questions. Taking into account that 62 questionnaire respondents agreed to identify themselves, the remaining 29 respondents provided center-focused data (e.g. the center location, the workload in the timeframe 2014 to 2018, and the number of gastroenterologist involved in the SBCE reading), which were so different that no duplicated data entries were disclosed; 91 centers were therefore included in the final analysis. Centers participating in the data collection were located in 17 of 20 Administrative Regions (85\%) (Appendix 2). SBCE was reimbursed as an outpatient procedure in 11 regions (55\% of regions; $69.3 \%$ of centers participating in the data collection) (Appendix 2), whereas patency capsule (PC) was not reimbursed in any of them. Thirty-six centers were academic (39.6\%), 49 were public hospitals (53.8\%) and 6 (6.6\%) were private hospitals that were part of the state-run health care system; no private hospitals outside the state-run health care system participated in the present survey. Most centers (95.6\%) performed SBCE examinations mainly in adults, whereas four centers were pediatric hospitals. The 91 centers participating in the data collection were equipped with 107 SBCE workstations, whereas PC and DAE were readily accessible in $63(69.2 \%)$ and 35 (38.5\%) of them, respectively. The geographical distribution of centers participating in the survey performing SBCE and DAE is represented in Appendix 2. SBCE was performed on a full open-access basis in $11.7 \%$ of centers, while in the remaining $88.3 \%$, an interview with a SBCE-dedicated gastroenterologist (who checked the patient's medical history, indication and contraindications) was required before receiving SBCE. Practical and procedural data collected in Section D are reported in Appendix 3.

In the 84 centers (92.3\%) that provided SBCE workload data, 16,535 examinations were performed in the 5-year timeframe from 2014 to 2018. The number of centers performing SBCE and the overall number of SBCEs per year are reported in - Fig. 1. In 2018, 3899 SBCEs were performed overall in 84 centers (median number of examinations per center: 46, IQR: 25-72, range: 3-142). Stratifying SBCEs performed in 2018, according to center type, 1618 SBCEs (41.5\%) were performed in 33 academic centers (median number of examinations per center: 48, IQR: 26-70, range: 12-142), whereas 2281 (58.5\%) in 51 non-academic centers (median number of examinations per center: 30, IQR: 20-61, range: 3-123). According to the 2018 caseload, 41 centers were defined as low-volume (median number of examinations per center: 21, IQR: $14-30$, range: $3-$ 35 ) and 43 as medium-high volume (median number of examinations per center: 63 , IQR: 48-84, range: 36-142) centers. In 2018 , nine centers (10.7\%) performed 614 (15.7\%) of SBCE on a full open access basis, whereas in the remaining 75 centers (89.3\%) 3285 (84.3\%) SBCEs were preceded by a clinical evaluation by a SBCE-dedicated gastroenterologist.

Sixty-two (68.1\%) questionnaire respondents agreed to identify themselves by answering the last question in Section C.

\section{Center policy concerning the measure of endoscopic procedures performances}

Fifty-one centers $(56.7 \%)$ participating in the data collection routinely monitored performance measures for non-SB procedures: in 36 (70.6\%) and 15 (29.4\%) cases at physician and service level, respectively. Before the publication of ESGE performance measures for small bowel endoscopy, 41 centers (45.0\%) already measured some self-established SBCE-related performance parameters, such as the spectrum of indications (36 of 41 centers, $87.8 \%$ ), the complication rate (23 centers, $56.1 \%$ ), the diagnostic yield (DY, 32 centers, $87.8 \%$ ), the rate of complete small bowel evaluation (28 centers, $78.0 \%$ ), the DAE referral rate ( 13 centers, $31.7 \%$ ) and the small bowel cleansing level ( 1 center, $2.4 \%)$.

\section{Performance measures of 2018 SBCEs}

More than $80 \%$ of centers reached the minimum standard established by the ESBWG in four of six key performance measures (the rate of correct indication for SBCE, the rate of complete small bowel evaluation, the lesion detection rate and the retention rate). Detailed results are reported in $\mathbf{\nabla}$ Table 2 . Concerning the remaining two key performance measures, the rate of patients with overt bleeding receiving SBCE within 14 days

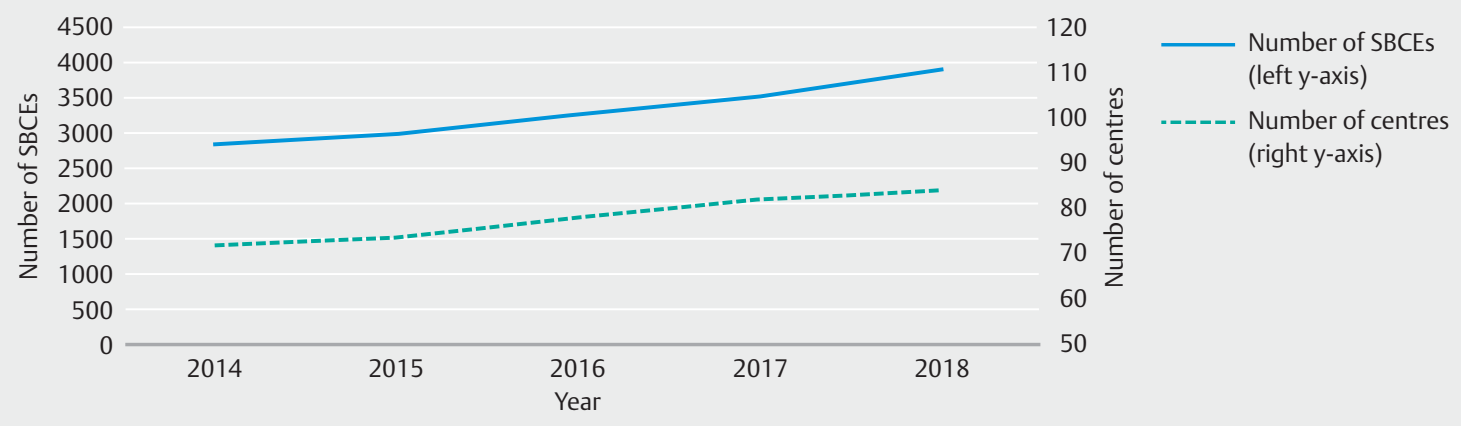

Fig. 1 Number of centers performing small bowel capsule endoscopy (SBCE) (left y-axis) and number of SBCEs performed (right y-axis) in the last five years (2014-2018). 
- Table 2 Rate of centers achieving the European Society of Gastrointestinal Endoscopy (ESGE) minimum and/or target standard of small bowel capsule endoscopy (SBCE) performance measures.

\begin{tabular}{|l|l|l|}
\hline Performance measure & $\begin{array}{l}\text { Rate of centers achievingng the ESGE } \\
\text { minimum standard }\end{array}$ & $\begin{array}{l}\text { Rate of centers achieving the ESGE target } \\
\text { standard }\end{array}$ \\
\hline Indication for SBCE & $80.3 \%$ & - \\
\hline Adequate bowel preparation & $15.5 \%$ & - \\
\hline Patient selection & $10.9 \%$ & $51.7 \%$ \\
\hline Caecal or stoma visualization & $89.1 \%$ & - \\
\hline Lesion detection rate & $80.0 \%$ & - \\
\hline Timing of SBCE for overt bleeding & $31.1 \%$ & - \\
\hline Use of standard terminology & $67.7 \%$ & $37.5 \%$ \\
\hline Reading speed of SBCE & $53.4 \%$ & $13.3 \%$ \\
\hline \begin{tabular}{l} 
Appropriate referral for DAE \\
\hline Capsule retention rate
\end{tabular} & $32.2 \%$ & - \\
\hline $\begin{array}{l}\text { Percentage in bold characters indicates whether performance measure minimum standards were met by more than } 80 \% \text { of centers. Minimun and Target standards } \\
\text { have the same threshold. } \\
\text { DAE, device-assisted enteroscopy. }\end{array}$ & $94.5 \%$ & \\
\hline
\end{tabular}

was $\geq 90 \%$ in $31.1 \%$ of centers and the rate of patients with positive SBCEs referred to DAE was $\geq 75 \%$ in $32.2 \%$ of centers ( Table 2).

None of the four minor performance measure minimum standards were achieved by a relevant proportion ( $\geq 80 \%$ ) of centers. In detail: the rate of patients with an adequate small bowel cleansing was $\geq 95 \%$ in $15.5 \%$ of centers, the rate of patients with high risk for capsule retention receiving PC was $\geq 95 \%$ in $10.9 \%$, the rate of SBCE reports written by using standard terminology was $\geq 90 \%$ in $67.7 \%$, and the rate of SBCE videos evaluated according to the reading speed suggested by ESBWG was $\geq 90 \%$ in $53.4 \%$ ( $\$$ Table 2 ). Variability across centers for both key and minor procedural quality performance measure is shown in Appendix 4.

- Table 3 summarizes the rate of centers reaching the minimum standards proposed by ESBWG, according to both the type of center and the 2018 caseload. No significant differences were found by comparing Academic and non-Academic Centers, whereas a significantly higher rate of patients receiving SBCE for an appropriate clinical indication was observed in low volume centers, when compared with medium-high volume centers (90.2\% vs. $72.1 \% ; P=0.05)$. Taking into account the very low number of centers performing SBCE on a full open-access basis and the lack of information about criteria applied in centers systematically evaluating patients before SBCE, we did not perform any comparison according to the policy for accessing the procedure.

\section{Discussion}

To the best of our knowledge, this is the first study comparing SBCE performance in everyday clinical practice with ESGE standards as quality benchmarks. This survey confirms that in Italy, capsule enteroscopy is a widespread diagnostic procedure with a constantly growing number of centers and examinations. The overall number of participating centers, their geographical distribution, and the overall number of procedures collected in this survey suggest that the present data might provide a reliable picture of SBCE performance on a nationwide basis. Interestingly, the percentage of centers (45\%) that had already been measuring some SBCE-related quality indicators, partially overlapping with those suggested by ESBWG, before specific indicators and standards were identified, as well as the publication of previous collaborative studies [19], confirms the clinical relevance of the topic and the interest of the Italian centers toward SBCE quality issues.

However, for the majority of the 10 SBCE performance measures, our data demonstrate wide variability across Italian centers. For four performance measures (indication for SBCE, complete small bowel evaluation, diagnostic yield and complication rate) the minimum standard established by ESBWG was met by more than $80 \%$ of the participating centers. Conversely, the minimum standard established by ESBWG for adequate bowel preparation, patient selection, timing of SBCE in overt bleeding, appropriate reporting, reading protocols and referral to DAE was reached by only $15.5 \%, 10.9 \%, 31.1 \%, 67.7 \%, 53.4 \%$ and $32.2 \%$ of centers, respectively.

Although the SBCE has been used in clinical practice for more than 20 years, the optimal preparation schedule is still being debated and recent evidence seems to question the schedule endorsed by guidelines $[20,21]$. This uncertainty may significantly contribute to variability across centers, which achieved the minimum standard in only $15 \%$ of cases, without significant differences, according to the caseload and the type of center. On one hand, this highlights the need to identify an effective preparation protocol that can be easily implemented in all centers. On the other hand, only one-quarter of centers participating in this survey used standardized scales to evaluate 
- Table 3 Rate of centers meeting the European Society of Gastrointestinal Endoscopy (ESGE) minimum of small bowel capsule endoscopy (SBCE) performance measures according to center type: (Academic vs. non-Academic) and center workload (low volume vs. high-volume) according to the definitions provided in the text.

\begin{tabular}{|c|c|c|c|c|c|c|}
\hline Performance measure & $\begin{array}{l}\text { Rate of Aca- } \\
\text { demic centers } \\
\text { achieving the } \\
\text { ESGE minimum } \\
\text { standard } \\
\text { ( } 33 \text { centers) }\end{array}$ & $\begin{array}{l}\text { Rate of non- } \\
\text { Academic cen- } \\
\text { ters achieving } \\
\text { the ESGE mini- } \\
\text { mum standard } \\
\text { (51 centers) }\end{array}$ & $P$ value $^{1}$ & $\begin{array}{l}\text { Rate of low vol- } \\
\text { ume }(\leq 35) \text { cen- } \\
\text { ters achieving } \\
\text { the ESGE mini- } \\
\text { mum standard } \\
\text { (41 centers) }\end{array}$ & $\begin{array}{l}\text { Rate of high- } \\
\text { volume ( } \geq 36 \text { ) } \\
\text { centers achiev- } \\
\text { ing the ESGE } \\
\text { minimum stand- } \\
\text { ard ( } 43 \text { centers) }\end{array}$ & $P$ value ${ }^{2}$ \\
\hline Indication for SBCE & $24 / 33(72.7)$ & $44 / 51(86.3)$ & 0.158 & $37 / 41(90.2)$ & $31 / 43(72.1)$ & 0.050 \\
\hline Adequate bowel preparation & $7 / 33(21.2)$ & $7 / 50(14.0)$ & 0.550 & $7 / 41(17.1)$ & $7 / 42(16.7)$ & 1.000 \\
\hline Patient selection & $4 / 33(12.2)$ & $4 / 51(7.8)$ & 0.706 & $5 / 41(12.2)$ & $3 / 43(7.0)$ & 0.478 \\
\hline Caecal or stoma visualization & 29/33 (87.9) & $46 / 51(90.2)$ & 0.733 & $38 / 41(92.7)$ & $37 / 43(86.1)$ & 0.484 \\
\hline Lesion detection rate & $26 / 32(81.2)$ & $40 / 51(78.4)$ & 1.000 & $33 / 40(82.5)$ & $33 / 43(76.7)$ & 0.592 \\
\hline Timing of SBCE for overt bleeding & $13 / 32(40.6)$ & $12 / 51(23.5)$ & 0.140 & $15 / 40(37.5)$ & $10 / 42(23.8)$ & 0.232 \\
\hline Use of standard terminology & $22 / 31(71.0)$ & $33 / 51(64.7)$ & 0.633 & $27 / 40(67.5)$ & $28 / 42(66.7)$ & 1.000 \\
\hline Reading speed of SBCE & $18 / 31(58.1)$ & $26 / 50(52.0)$ & 0.651 & $18 / 38(47.4)$ & $26 / 42(61.9)$ & 0.261 \\
\hline Appropriate referral for DAE & $9 / 32(28.1)$ & $17 / 51(33.3)$ & 0.808 & $11 / 40(27.5)$ & $15 / 43(34.9)$ & 0.171 \\
\hline Capsule retention rate & $30 / 32(93.7)$ & $48 / 51(94.1)$ & 1.000 & $38 / 40(95.0)$ & $40 / 43(93.0)$ & 1.000 \\
\hline
\end{tabular}

small bowel cleansing. As recommended by ESGE technical guidelines [19] cleansing scales and scores are helpful in standardizing the SBCE report, thus avoiding long, arbitrary, and sometimes ambiguous descriptions. Interestingly the low rate of centers achieving adequate bowel preparation in more than $95 \%$ of patients somewhat conflicts with the high percentage ( $80 \%$ ) of centers where a diagnostic yield higher than $50 \%$ was observed. Although the diagnostic performance may be influenced by several factors, small bowel cleansing plays a major role. The observed discrepancy between diagnostic yield and adequate cleansing rate contributes to challenging the ESGE standards, which are established by consensus and often based on limited low-quality evidence [13]. Concerning the rate of adequate small bowel preparation, if the minimum standard was established at $75 \%$ (instead of $95 \%$ ), the rate of centers meeting this threshold would rise to over $70 \%$ (71.1\%), which appears more consistent with the rate of Italian centers having a high diagnostic yield.

Despite current clinical guidelines that recommend performing SBCE within 14 days in patients with overt bleeding [22], this issue appears to have wide variability among Italian centers. Of note, the rate of centers performing SBCE within 14 days remains suboptimal, even in academic or high-volume centers. Patients with overt bleeding are usually evaluated in an Emergency Room (ER) setting, where the gastroenterologist is seldom involved in their management. The importance of including an early SBCE in the diagnostic process needs to be emphasized and widespread, mainly among non-gastroenterologists. There is growing evidence supporting the very early use of SBCE, even in the ER setting [23-26], in patients with overt bleeding. Conversely, at present, once the acute event has resolved, these patients are usually referred to the gastroenterologist through an outpatient evaluation, which often has a long waiting list. It might be appropriate to set up a preferential fast track with early involvement of the gastroenterologist, maybe in the ER, to plan a timely SBCE, if needed.

The high rate of centers (about $90 \%$ ) in which a SBCE-dedicated gastroenterologist carries out a clinical evaluation before performing SBCE might have contributed to the high diagnostic yield and to the high rate of appropriate SBCE observed in the present survey. However, this effective pre-procedural filter appears to conflict with the low percentage of patients with high risk of device retention who systematically receive PC, which is available in $70 \%$ of centers. In everyday clinical practice, many patients with clinical conditions, such as known Crohn's disease, obstructive symptoms, and suspected small bowel tumors, have a significant risk of small bowel stenosis and are often referred to SBCE after receiving negative small bowel-dedicated cross-sectional imaging. This is readily available, reimbursed everywhere, and generally considered reliable in ruling out the risk of capsule retention.

Nevertheless, cross-sectional imaging is less accurate in the evaluation of functional small bowel patency and frequently overestimates the risk of obstruction $[27,28]$. Screening of small bowel patency by PC, therefore, may be implemented by introducing reimbursement for PC, but also by improving and standardizing the PC test process, since checking for a capsule 
in feces is unpleasant for the patient and a precise PC location might still require computed tomography scanning $[29,30]$.

In the present survey, the rate of centers referring $75 \%$ or more of patients with positive SBCE for DAE is surprisingly low (32\%). This figure might result from several factors. On one hand, some SBCE positive findings might have limited clinical relevance and/or do not necessitate subsequent DAE, such as multiple non-bleeding small bowel angioectasias, which might initially be managed conservatively with iron supplementation. On the other hand, this could be also related to the limited availability of centers performing DAE in Italy. According to the collected data, fewer than $40 \%$ of centers performing SBCE are also performing DAE and their geographical location appears to be inhomogeneous, with five regions in which there were nine centers performing SBCE overall but no centers performing DAE, and definitely suboptimal.

The present survey has some limitations. First, the study was observational, and the evaluation of performance parameters was retrospective. Therefore, reported percentages, although based on several consecutive examinations in a well-defined timeframe, represent estimations more than precise measurements. Unfortunately, some parameters we evaluated, such as reading speed, are not systematically included in the SBCE reports; therefore, the answer provided was most likely to reflect the reader's usual policy rather than actual data. In addition, the descriptive nature of the survey did not provide for any explanation regarding the observed variation. To fully address this issue, a questionnaire with long list of additional questions (definitely longer and more detailed than Section D) focused on several practical, technical and organizational issues in each center should be planned. The knowledge of these data, along with a better dissemination of ESGE standards and a well-established accreditation path, would be helpful to improve adherence to ESGE guidelines and to optimize SBCE in clinical practice. Second, as in every survey, a selection bias cannot be fully ruled out; nevertheless, the high number of participating centers and their geographical distribution might contribute to reducing the relevance of the possible selection bias. Third, some parameters were difficult to check, such as the adoption of a reading protocol in line with ESGE recommendations and the exact time between the overt bleeding episode and SBCE, and although the questionnaire mirrored the original ESGE document, it was semi-quantitative. On one hand, this allowed for collection of reliable data anonymously, quickly, and easily, without consulting hospital databases for most of the topics. On the other hand, the data lacked precision and might have increased the risk of biases, such as recall or estimator bias. Fourth, the questionnaire was self-reported; even if that facilitated the participation of centers in data collection, it introduced a potential response bias. Response bias is a widely discussed phenomenon in research fields (such as behavioral and health care research) where self-reported data are used [31]. It occurs when individuals offer self-assessed measures of some phenomenon and a respondent wants to look good in the survey, even if the survey is anonymous, or when there is a misunderstanding about what a proper measurement is. Last but not least, to facilitate the data analysis and results interpretation, some arbitrary thresholds were established, such as, if more than $80 \%$ of centers reached the ESGE minimum standard, we assumed that the variability across centers was low and centers were classified as medium-high volume if more than 35 procedures were performed in 2018.

\section{Conclusion}

In conclusion, this data collection, involving 91 centers, clearly demonstrates that although SBCE is widely used and largely available in Italy, the performance of this procedure in clinical practice is extremely variable and still suboptimal in many centers. We, therefore, focused on aspects where wider variability was observed, because they are target areas for corrective measures and for further quality improvement programs in SBCE. This data collection is the first attempt to evaluate SBCE performance in light of standardized expert consensus-based thresholds. Unfortunately, mostly because of its descriptive nature, it brings to our attention more questions than answers. Therefore, international prospective studies, covering a longer timeframe, merging a questionnaire on performance with more detailed center-focused data and involving a higher number of centers and countries, are warranted. They would be also helpful to validate the ESBWG quality standard thresholds in order to optimize the quality improvement process in the field of SBCE.

\section{Acknowledgments}

The authors thank Medtronic Italia (namely M. Ferrari, M. Mozzi, and U. Pagliusi, as well as all the local agents and distributors) for the continuous logistic support. Medtronic (Medtronic Italia S.p.A., Milano, Italy) provided unconditional logistic support for the data collection and selection of the web-based platform.

\section{RAVE Study Group (in alphabetical order):}

Stefano Ambrogio Bargiggia, Alessandra Bizzotto, Valentina Boarino, Fabrizio Bossa, Rosamaria Bozzi, Mauro Bruno, Andrea Buda, Marisa Buono, Danilo Castellani, Flaminia Cavallaro, Linda Ceccarelli, Laura Centenara, Paola Ciamarra, Giovanni Comi, Debora Compare, Giovanna Condino, Claudio Camillo Cortelezzi, Marco Daperno, Elena Di Girolamo, Kostantinos Efthymakis, Maurizio Ferrante, Fabiola Fornaroli, Giusi Franco, Tommaso Gabbani, Ermenegildo Galliani, Carlotta Francesca Maria Gebbia, Tiziana Grasso, Elena liritano, Paulin Francis Lawson, Fausto Lella, Eugenio Limido, Marta Maino, Giovanna Mandelli, Nicola Mantovani, Marco Marino, Roberta Marino, Mauro Mastronardi, Dario Moneghini, Elisabetta Morandi, Alessandro Mussetto, Chiara Notaristefano, Maurizio Pantalena, Carmelina Petruzziello, Lorena Pietrini, Lodovica Pozzi, Mariabeatrice Prinicipi, Rossella Pumpo, Maria Luisa Rega, Stefano Rodinò, Renzo Schalling, Clementina Signorelli, Maria Giuseppina Tatarella, Gian Eugenio Tontini, Filippo Torroni, Pasquale Vitagliano, Giovanna Vitale. 


\section{Competing interests}

Dr. Spada is consultant for Medtronic and Norgine and received grant from AlfaSigma, Pentax, Olympus. Dr.Pennazio received speaker's honoraria from Medtronic and Olympus. Dr. Rondonotti received speaker's honoraria from Fujifilm.

\section{References}

[1] Sapienza PE, Levine GM, Pomerantz $S$ et al. Impact of a quality assurance program on gastrointestinal endoscopy. Gastroenterology 1992; 102: 387-393

[2] Corley DA, Jensen CD, Marks AR et al. Adenoma detection rate and risk of colorectal cancer and death. N Engl J Med 2014; 370: 12981306

[3] Yalamarthi S, Witherspoon P, McCole D et al. Missed diagnoses in patients with upper gastrointestinal cancers. Endoscopy 2004; 36: 874879

[4] Raftopoulos SC, Segarajasingam DS, Burke $V$ et al. A cohort study of missed and new cancers after esophagogastroduodenoscopy. Am J Gastroenterol 2010; 105: 1292-1297

[5] Enochsson L, Swahn F, Arnelo U et al. Nationwide, population-based data from 11,074 ERCP procedures from the Swedish Registry for Gallstone Surgery and ERCP. Gastrointest Endosc 2010; 72: 11751184

[6] Cohen J, Pike IM. Defining and measuring quality in endoscopy. Gastrointest Endosc 2015; 81: 1-2

[7] Rutter MD, Senore C, Bisschops R et al. The European Society of Gastrointestinal Endoscopy Quality Improvement Initiative: developing performance measures. Endoscopy 2016; 48: 81-89

[8] Rees C], Thomas Gibson S, Rutter MD et al. UK key performance indicators and quality assurance standards for colonoscopy. Gut 2016; 65: $1923-1929$

[9] Valori R, Cortas G, de Lange T et al. Performance measures for endoscopy services: a European Society of Gastrointestinal Endoscopy (ESGE) Quality Improvement Initiative. Endoscopy 2018; 50: 1186-1204

[10] Bisschops R, Areia M, Coron E et al. Performance measures for upper gastrointestinal endoscopy: a European Society of Gastrointestinal Endoscopy (ESGE) Quality Improvement Initiative. Endoscopy 2016; 48: 843-864

[11] Domagk D, Oppong KW, Aabakken L et al. Performance measures for ERCP and endoscopic ultrasound: a European Society of Gastrointestinal Endoscopy (ESGE) Quality Improvement Initiative. Endoscopy 2018; 50: 1116-1127

[12] Kaminski MF, Thomas-Gibson S, Bugajski M et al. Performance measures for lower gastrointestinal endoscopy: a European Society of Gastrointestinal Endoscopy (ESGE) Quality Improvement Initiative. Endoscopy 2017; 49: 378-397

[13] Spada C, McNamara D, Despott E] et al. Performance measures for small-bowel endoscopy: a European Society of Gastrointestinal Endoscopy (ESGE) Quality Improvement Initiative. United European Gastroenterol J 2019; 7: 21-44

[14] Albert JG, Humbla O, McAlindon ME et al. A simple evaluation tool (ET-CET) indicates increase of diagnostic skills from small bowel capsule endoscopy training courses: a prospective observational European multicenter study. Medicine (Baltimore) 2015; 94: e1941
[15] Rajan E, Martinez M, Gorospe E et al. Prospective multicenter study to evaluate capsule endoscopy competency using a validated as- sessment tool. Gastrointest Endosc 2020; 91: 1140-1145

[16] The European Section and Board of Gastroenterology and Hepatology. Speciality Training Programme and Curriculum for Gastroenterology and Hepatology. The Blue Book; 2017: Available from (last accessed 09/08/2020): http://www.eubogh.org/blue-book/

[17] Rajan E, lyer PG, Oxentenko AS et al. Training in small-bowel capsule endoscopy: assessing and defining competency. Gastrointest Endosc 2013; 78: 617-622

[18] Sidhu R, Chetcuti Zammit S, Baltes P et al. Curriculum for small-bowe capsule endoscopy and device assisted enteroscopy training in Europe: European Society of Gastrointestinal Endoscopy (ESGE) Position statement. Endoscopy 2020; 52: 669-686

[19] Rondonotti E, Spada C, Pennazio M et al. Adherence to European Society of Gastrointestinal Endoscopy recommendations of endoscopists performing small bowel capsule endoscopy in Italy. Dig Liver Dis 2019; 51 : 818-823

[20] Rondonotti E, Spada C, Adler S et al. Small-bowel capsule endoscopy and device-assisted enteroscopy for diagnosis and treatment of small-bowel disorders: European Society of Gastrointestinal Endoscopy (ESGE) Technical Review. Endoscopy 2018; 50: 423-446

[21] Gkolfakis P, Tziatzios G, Dimitriadis GD et al. Meta-analysis of randomized controlled trials challenging the usefulness of purgative preparation before small-bowel video capsule endoscopy. Endoscopy 2018; 50: 671-683

[22] Pennazio M, Spada C, Eliakim R et al. Small-bowel capsule endoscopy and device-assisted enteroscopy for diagnosis and treatment of small-bowel disorders: European Society of Gastrointestinal Endoscopy (ESGE) Clinical Guideline. Endoscopy 2015; 47: 352-376

[23] Yung DE, Koulaouzidis A, Douglas $S$ et al. Earlier use of capsule endoscopy in inpatients with melena or severe iron deficiency anemia reduces need for colonoscopy and shortens hospital stay. Endosc Int Open 2018; 6: E1075-E1084

[24] Marya NB, Jawaid S, Foley A et al. A randomized controlled trial comparing efficacy of early video capsule endoscopy with standard of care in the approach to nonhematemesis GI bleeding (with videos). Gastrointest Endosc 2019; 89: 33-43

[25] lio S, Oka S, Tanaka S et al. Clinical utility of emergency capsule endoscopy for diagnosing the source and nature of ongoing overt obscure gastrointestinal bleeding. Gastroenterol Res Pract 2019: doi: $10.1155 / 2019 / 5496242$

[26] Schlag C, Menzel C, Nennstiel S et al. Emergency video capsule endoscopy in patients with acute severe Gl bleeding and negative upper endoscopy results. Gastrointest Endosc 2015; 81: 889-895

[27] Rozendorn N, Klang E, Lahat A et al. Prediction of patency capsule retention in known Crohn's disease patients by using magnetic resonance imaging. Gastrointest Endosc 2016; 83: 182-187

[28] Rondonotti E, Soncini M, Girelli CM et al. Short article: Negative smallbowel cross-sectional imaging does not exclude capsule retention in high-risk patients. Eur J Gastroenterol Hepatol 2016; 28: 871-875

[29] Omori T, Nakamura S, Shiratori K. Localization of the patency capsule by abdominal tomosynthesis. Digestion 2015; 91: 318-325

[30] Assadsangabi A, Blakeborough A, Drew K et al. Small bowel patency assessment using the patency device and a novel targeted (limited radiation) computed tomography-based protocol. J Gastroenterol Hepatol 2015; 30: 984-989

[31] Tennekoon G, Hill LG. Measuring bias in self-reported data. Int J Behav Healthc Res 2011; 2: 320-332 